\title{
Inventário de Expectativas de Resultados em Usuários de Maconha (IERUM): construção e validação
}

\author{
Inventory of Outcome Expectancy in Marijuana Users (Inventário de Expectativas de \\ Resultados em Usuários de Maconha, IERUM): development and validation
}

\author{
Rosemeri Siqueira Pedroso', Maria da Graça Tanori de Castro ${ }^{2}$, Renata Brasil Araujo
}

${ }^{1}$ Mestre, Psicologia Clínica, Pontifícia Universidade Católica do Rio Grande do Sul (PUCRS), Porto Alegre, RS. XXX, Universidade Federal do Rio Grande do Sul (UFRGS), Porto Alegre, RS. ${ }^{2}$ Mestre, Psicologia Clínica PUCRS. Professora, Curso de Pós-Graduação lato sensu, XXX, Instituto Abuchaim, Porto Alegre, RS. ${ }^{3}$ Doutora, Psicologia, PUCRS. Coordenadora, Programa de Dependência Química, Hospital Psiquiátrico São Pedro, Porto Alegre, RS.

Este trabalho foi realizado no Hospital Psiquiátrico São Pedro, Porto Alegre, RS.

\section{Resumo}

Introdução: Este estudo apresenta a construção e validação do Inventário de Expectativas de Resultados em Usuários de Maconha (IERUM), o qual avalia as expectativas de resultados em relação ao uso de maconha, podendo ser importante no tratamento e prognóstico dos dependentes químicos dessa substância.

Método: $\mathrm{O}$ estudo foi realizado em uma amostra de 181 sujeitos usuários de maconha, homens $(\mathrm{n}=145)$ e mulheres $(\mathrm{n}=36)$, entre 18 e 55 anos (28,34 $\pm 8,57)$; 118 estavam internados em unidade de dependência química, 23 em tratamento ambulatorial, 25 em grupo de autoajuda (narcóticos anônimos, alcoólatras anônimos) e 15 não estavam em tratamento. A escala de 17 itens foi criada a partir da experiência em dependência química e em validação de escalas, sendo realizada primeiramente a validação semântica.

Resultados: De acordo com a análise fatorial, o IERUM dividiu-se em cinco fatores (aspectos emocionais, percepção, craving, aspectos cognitivos e sexualidade) que responderam por $60,18 \%$ da variância.

Conclusão: O IERUM apresentou bons resultados psicométricos, podendo avaliar as expectativas de resultados relacionadas ao uso de maconha. Descritores: Cannabis, expectativas, IERUM, construção, validação.

\begin{abstract}
Introduction: This study presents the development and validation of the Inventory of Outcome Expectancy in Marijuana Users (Inventário de Expectativas de Resultados em Usuários de Maconha, IERUM), which evaluates the outcome expectancy regarding marijuana use, being useful in the treatment and prognosis of marijuana-dependent individuals.

Method: The sample comprised 181 male $(n=145)$ and female $(n=36)$ marijuana users. Their age ranged from 18 to 55 years old $(28.34 \pm 8.57)$. One hundred and eighteen patients were hospitalized at a chemical dependency unit, 23 were being treated in an outpatient clinic, 25 were receiving treatment in a self-help group (drug addicts anonymous, alcoholic anonymous), and 15 were not being treated. The 17-item scale was developed based on the experience related to chemical dependency and scale validation, with the semantic validation being the first phase of the process.

Results: According to the factor analysis, the IERUM was divided into five factors (emotional aspects, perception, craving, cognitive aspects, and sexual aspects) that accounted for $60.18 \%$ of the variance.

Conclusion: The IERUM showed good psychometric properties, being useful to evaluate the outcome expectancy related to marijuana use.

Keywords: Cannabis, expectancy, IERUM, development, validation.
\end{abstract}

\section{Correspondência:}

Maria da Graça Tanori de Castro, Rua Mariante, 288/407,

CEP 90430-180, Porto Alegre, RS. Praça Conde de Porto Alegre, 22/303, Bairro Centro, CEP 90.020-130, Porto Alegre, RS. Tel.: (51) 3222.1154, (51) 3227.3097, (51) 9983.8430, (51) 9997.2435 E-mail: mgdc@uol.com.br

Não há conflitos de interesse associados à publicação deste artigo

Copyright (C) Revista de Psiquiatria do Rio Grande do Sul - APRS

Recebido em 28/06/2009. Aceito em 02/10/2009. 


\section{Introdução}

As expectativas de resultados em relação aos efeitos de uma substância psicoativa podem desencadear o comportamento aditivo, levando o sujeito a decidir-se pelo uso dessa substância devido à antecipação do prazer associado ao seu consumo. A cognição, a cultura e a personalidade influenciam nas crenças acerca dos efeitos de determinada droga, fazendo com que o sujeito ingresse no comportamento dependente quando ele relaciona suas experiências aos efeitos do uso de substâncias psicoativas ${ }^{1,2}$. As informações obtidas nos meios de comunicação, com amigos e com figuras parentais a respeito de substâncias psicoativas podem levar o sujeito a observar e aprender comportamentos, acreditando que essas substâncias se associam a resultados positivos ou negativos ${ }^{3,4}$.

$\mathrm{Na}$ clínica de dependência química, as expectativas de resultados positivos devem ser examinadas com cuidado no processo de recaída, pois a crença que o sujeito tem acerca dos efeitos de determinada substância psicoativa pode desencadear um comportamento de busca pelo consumo da mesma, independentemente de que os efeitos reais da droga sejam muito diferentes dos efeitos esperados. Já nos anos 1980, alguns teóricos consideraram o construto "expectativa de resultado" um mediador cognitivo do comportamento, especificamente para o consumo e dependência de drogas ${ }^{5}$. Dada a relevância desse tema, torna-se importante desenvolver e validar um instrumento que mensure, especificamente, as expectativas de resultados diante do uso das drogas, dentre elas, a maconha ${ }^{2,6}$.

Hawkins ${ }^{7}$ testou uma série dos modelos mediadores do relacionamento entre as variáveis afetivas relacionadas à personalidade e o uso de maconha em uma amostra de estudantes universitárias que tinham usado maconha ao menos uma vez na vida. Os resultados demonstraram elevada aflição emocional e psicológica e índices elevados de expectativas de relaxamento e redução de tensão significativamente relacionados ao uso de maconha. Também apareceram expectativas de resultados associando o uso de maconha à abertura de consciência, realçando a percepção e a cognição. Os níveis mais elevados de expectativas percebidas em relação ao uso de maconha foram os relativos às expectativas de facilitação social e sexual. O estudo também sugeriu que os aspectos emocionais e psicológicos predizem a conformidade para o uso de maconha.

As implicações do uso de maconha em adolescentes que estavam em tratamento foram avaliadas em 82 adolescentes que participaram de outro estudo e que apresentavam taxas elevadas de uso de álcool, maconha e nicotina. O uso de maconha e nicotina estava associado a taxas elevadas de sintomas depressivos. Os adolescentes esperavam que a maconha tivesse um impacto positivo maior e um impacto negativo muito pequeno em suas vidas, não se mostrando confiantes em suas habilidades de resistir ao uso da maconha, embora muitos demonstrassem interesse em operar mudanças nesse sentido ${ }^{8}$.

No Brasil, para avaliar as expectativas de resultado frente ao uso da maconha, foi validado o Marijuana Expectancy Questionnaire (MEQ) ${ }^{3}$. A amostra, por conveniência, foi de
400 sujeitos, homens $(n=217)$ e mulheres $(n=183)$, entre 12 e 60 anos de idade, usuários de maconha $(n=147)$ e não usuários $(\mathrm{n}=253)$. Dentre os usuários, 90 eram dependentes de maconha e, deles, 56 estavam internados. O coeficiente de Cronbach foi de 0,89 para a escala com 78 itens e de 0,89 para a escala reduzida, com 55 itens. Nessa amostra brasileira, aplicaram-se os 78 itens, porém a análise estatística foi realizada a partir da escala reduzida, pois as propriedades psicométricas foram similares. A validade discriminante para a escala reduzida foi estatisticamente significativa. A estabilidade temporal foi examinada em uma amostra de 123 sujeitos, com um intervalo de 1 semana entre o teste e o reteste, indicando um padrão consistente de resposta ao longo do tempo. Como muitos itens da escala tiveram que ser excluídos - e, ainda assim, a escala permaneceu longa - e não foi possível realizar a análise fatorial devido às limitações psicométricas da própria escala, foi elaborada uma nova escala para avaliar o construto expectativa de resultado frente ao uso da maconha, o Inventário de Expectativas de Resultados em Usuários de Maconha (IERUM).

Para a construção de um instrumento de medida são necessárias as definições constitutivas que irão contextualizar o construto alvo dentro da teoria e da literatura disponível e estudada. Nem sempre um instrumento que mede um construto abrangerá toda a amplitude semântica de um conceito. Neste estudo do IERUM, priorizam-se as boas definições constitutivas que permitem avaliar a qualidade do inventário, pois o processo de construção e validação de um instrumento de pesquisa deve ser criterioso, e a equivalência conceitual deve ser obtida através do brainstorming e da análise dos juízes ${ }^{9-11}$.

Assim, o objetivo deste trabalho é apresentar a construção e validação semântica e psicométrica do IERUM.

\section{Método}

\section{Delineamento e participantes}

Foi realizado um estudo transversal. O estudo foi realizado em uma amostra por conveniência de 181 sujeitos. Deles, 150 eram dependentes de maconha $(82,87 \%)$ e 31 faziam uso nocivo dessa substância $(17,13 \%)$, de acordo com a CID-10 ${ }^{12}$. Os participantes eram homens $(\mathrm{n}=145)$ e mulheres $(\mathrm{n}=36)$, entre 18 e 55 anos $(28,34 \pm 8,57) ; 118$ estavam internados em unidade de dependência química, 23 em tratamento ambulatorial, 25 em grupo de autoajuda (narcóticos anônimos, alcoólatras anônimos) e 15 não estavam em tratamento. A escolaridade mínima exigida foi a $5^{\mathrm{a}}$ série do Ensino Fundamental. Foram excluídos do estudo os sujeitos que não possuíam a escolaridade mínima exigida, assim como os sujeitos com qualquer comprometimento cognitivo que impedisse a compreensão e o preenchimento dos instrumentos.

Foram observados todos os princípios éticos envolvidos nas pesquisas com seres humanos, sendo que o projeto foi aprovado pelo Comitê de Ética do Hospital Psiquiátrico São Pedro, Porto Alegre (RS). 


\section{Instrumentos}

- Entrevista estruturada com o objetivo de definir o perfil sociodemográfico da amostra estudada, os diagnósticos relacionados ao uso de substâncias psicoativas utilizando critérios da Classificação Estatística Internacional de Doenças e Problemas Relacionados à Saúde (CID-10) ${ }^{12}$ e a história de consumo de maconha e das outras drogas.

- IERUM: uma escala Likert estruturada, um tipo de escala de resposta psicométrica usada comumente em questionários ${ }^{13}$, com 17 itens relacionados ao uso de maconha nos quais são atribuídos pontos de 1 (discordo totalmente) a 7 (concordo totalmente), distribuídos em cinco subescalas: aspectos emocionais, percepção, sexualidade, aspectos cognitivos e craving.

- Escalas analógico-visuais (EAV): as EAV são amplamente utilizadas em pesquisa ${ }^{14-16}$. Neste estudo, foram usadas cinco escalas que avaliam aspectos emocionais, percepção, sexualidade, aspectos cognitivos e craving.

\section{Procedimentos}

\section{Construção do IERUM}

O IERUM foi criado com base nas seguintes categorias definidoras deste construto: aspectos emocionais, percepção, craving, aspectos cognitivos e sexualidade. A escolha dessas categorias foi feita a partir de quesitos avaliados em estudos relacionados a expectativas de resultado frente ao uso de maconha ${ }^{6-8}$. Os passos seguidos para a construção da escala foram: (a) estudo das dimensões de conteúdo das subescalas para a definição da matriz de conteúdo; (b) criação, pelas autoras, de 18 itens para a escala a partir das definições e da análise da literatura sobre o construto e da análise de outro instrumento anteriormente validado no Brasil ${ }^{6}$; (c) discussão para o julgamento e a seleção de itens com base nos critérios de clareza, precisão de linguagem, simplicidade e adequabilidade da classificação em relação aos conceitos; (d) organização aleatória dos itens em afirmativas descritivas de experiências ligadas às expectativas de resultados em relação ao uso de maconha por meio de uma escala Likert de 1 (discordo totalmente) a 7 (concordo totalmente); (e) revisão da redação dos itens da escala por um especialista em língua portuguesa; (f) estudo piloto com 30 sujeitos usuários de maconha para avaliação da compreensão das instruções e dos itens; (g) brainstorming: uma técnica cuja tradução é "tempestade de ideias". Trata-se de uma metodologia de exploração de ideias visando à obtenção das melhores soluções de um grupo de pessoas ${ }^{17}$. Neste estudo, 3 psicólogos especialistas em dependência química discutiram acerca da clareza e relevância dos itens, revisão, redação e editoração final. A escala final foi composta por 17 itens, sendo observados os critérios para a construção de escalas descritos por Pasquali ${ }^{9}$.

\section{Coleta dos dados}

A versão final da escala foi aplicada de forma individual e preenchida pelo próprio participante após a assinatura do Termo de Consentimento Livre e Esclarecido para participa- ção no estudo mediante orientação quanto aos objetivos da pesquisa, uso a ser dado às informações obtidas e garantia de sigilo. A fase de coleta de dados ocorreu durante o período de janeiro de 2007 a julho de 2008 .

\section{Análise dos dados}

As informações coletadas foram organizadas no Programa Statistical Package for the Social Sciences (SPSS Inc., Chicago, EUA) versão 16.0. A análise exploratória dos dados constou de testes estatísticos descritivos e de frequências. $\mathrm{Na}$ validação do MEQ, foram utilizados o $\alpha$ de Cronbach para verificar a consistência interna do instrumento e o coeficiente de correlação linear de Pearson para verificar a estabilidade temporal do instrumento. Para avaliar se as variáveis tinham uma distribuição normal, foi utilizado o teste KolmogorovSmirnov. A análise inferencial empregou os testes: teste Mann-Whitney, análise fatorial com rotação Promax e coeficiente de correlação linear de Pearson. Com o objetivo de comprovar a adequação dos dados do IERUM para a análise fatorial foi utilizado o Kaiser-Meyer-Olkin (KMO) e o teste de Bartlett. O nível de significância utilizado foi o de 5\%.

\section{Resultados}

\section{Perfil sociodemográfico}

Da amostra total $(\mathrm{n}=181), 150$ sujeitos eram dependentes de maconha $(82,87 \%)$ e 31 faziam uso nocivo dessa substância $(17,13 \%)$. Os participantes usavam uma média de 23,41 baseados por semana [desvio padrão $(\mathrm{DP})=32,62 ; 1-210$ ]

Os demais dados sociodemográficos e referentes ao padrão de uso de substâncias psicoativas podem ser observados na Tabela 1 .

Tabela 1 - Dados sociodemográficos e referentes ao padrão de uso de substâncias psicoativas $(n=180)$

\begin{tabular}{lc}
\hline Variáveis & n (\%) \\
\hline Escolaridade & \\
Até 4 anos de estudo & $17(9,4)$ \\
Entre 5 e 8 anos de estudo & $71(39,2)$ \\
Ensino médio incompleto & $33(18,2)$ \\
Ensino médio completo & $22(12,2)$ \\
Ensino superior incompleto & $28(15,5)$ \\
Ensino superior completo & \\
Missing = & \\
Estado Civil & \\
Solteiros & $5(2,8)$ \\
Casados & $141(77,9)$ \\
Separados & $22(12,2)$ \\
Viúvos & $7(7,7)$ \\
Missing = & $2(1,1)$ \\
Dependência de outras substâncias psicoativas & \\
Alcool & $74(40,7)$ \\
Cocaína inalada & $137(75,3)$ \\
Crack & $134(73,6)$ \\
Nicotina & $156(85,7)$ \\
Mais de uma substância psicoativa & $166(91,2)$ \\
Só a nicotina além da maconha & $38(20,99)$ \\
Usavam somente a maconha & $7(3,87)$ \\
\hline
\end{tabular}




\section{Validação semântica}

Para a construção do IERUM, utilizou-se o critério de decisão sobre a pertinência do item conforme Pasquali ${ }^{9}$.

Na validação semântica do IERUM, seguiram-se os passos propostos por Pedroso et al. ${ }^{10}$.

\section{Versão final do instrumento}

Inicialmente, o IERUM constituiu-se em uma escala de 18 itens; no entanto, um item foi excluído (questão 4) a partir da análise fatorial. Os itens restantes foram distribuídos em cinco subescalas: I) aspectos emocionais (questões 7, 14, 15, 16 e 18); II) percepção (questões 2, 3, 5, 6 e 12); III) sexualidade (questões 9 e 17); IV) aspectos cognitivos (questões 8, 11 e 13); e V) craving (questões 1 e 10).

\section{Consistência interna}

A fidedignidade estimada, baseada no coeficiente de Cronbach, na escala total de 17 itens, foi de 0,779 .

Os valores do coeficiente de Cronbach nas cinco subescalas são: aspectos emocionais, 0,42 $(\mathrm{n}=5)$; percepção, 0,67 $(\mathrm{n}=5)$; sexualidade $0,83(\mathrm{n}=2)$; aspectos cognitivos, 0,69 $(\mathrm{n}=3)$; e craving, 0,69 $(\mathrm{n}=2)$.

\section{Análise fatorial}

O resultado do KMO foi de 0,74 e o do teste de Bartlett, $p$ $<0,001$, demonstrando que a utilização da análise de variância (ANOVA) seria adequada para a validação desta escala.

Segue, na Tabela 2, a distribuição das questões nos fatores e categorias da escala a partir da utilização da rotação Promax. Não foi predeterminado estatisticamente o número de fatores pelas pesquisadoras, sendo a solução de cinco fatores o resultado encontrado.
Foi utilizado o critério de Tiffany \& Drobes ${ }^{18}$ para a colocação das questões nos fatores, considerando-se como pertencentes ao fator itens com carga fatorial $\geq 0,40$ cuja carga no outro fator fosse $<0,25$, devendo a diferença entre ambas ser de, pelo menos, 0,20.

O fator I (aspectos emocionais) teve autovalor de 4,06 e variância de 22,58\%; o fator II (percepção) teve autovalor de 3,04 e variância de 16,91\%; o fator III (sexualidade) teve autovalor de 1,41 e variância de 7,84\%; o fator IV (aspectos cognitivos) teve autovalor de 1,22 e variância de 6,78\%; e o fator V (craving) teve autovalor de 1,09 e variância de $6,07 \%$. O total da variância foi equivalente a $60,19 \%$.

A questão 4 foi excluída por não preencher o critério de Tiffany \& Drobes ${ }^{18}$ para a colocação das questões nos fatores, ficando, a versão final do IERUM com 17 itens.

\section{Validade de critério}

A validade de critério foi medida correlacionando-se as subescalas entre si por meio do coeficiente de correlação linear de Pearson, sendo que todos os resultados foram abaixo de 0,80 , indicando não haver redundância, conforme preconizam Ferrans \& Powers ${ }^{19}$. Os achados encontram-se na Tabela 3.

\section{Validade convergente}

A validade convergente foi avaliada aplicando o coeficiente de correlação linear de Pearson entre as subescalas do IERUM e as EAV, sendo os resultados apresentados na Tabela 4.

\section{Validade discriminante}

A validade discriminante foi mensurada por meio do teste Mann-Whitney, comparando as subescalas do IERUM entre os indivíduos dependentes e os que faziam uso nocivo de maconha. Os resultados são apresentados na Tabela 5.

Tabela 2 - Análise fatorial com a rotação Promax

\begin{tabular}{|c|c|c|c|c|c|}
\hline \multirow[b]{2}{*}{ Questões do IERUM } & \multicolumn{5}{|c|}{ Fatores } \\
\hline & $\begin{array}{c}\text { Aspectos } \\
\text { emocionais }\end{array}$ & Percepção & Sexualidade & $\begin{array}{c}\text { Aspectos } \\
\text { cognitivos }\end{array}$ & Craving \\
\hline 1. Tenho fissura (desejo intenso) por maconha & & & & & 0,896 \\
\hline 2. Fujo da realidade quando fumo maconha & & 0,664 & & & \\
\hline 3. Tudo fica mais interessante quando fumo maconha & & 0,400 & & & \\
\hline 4. Maconha deixa-me mais sociável* & & 0,405 & & -473 & \\
\hline 5. Fico rindo à toa quando fumo maconha & & 0,648 & & & \\
\hline 6. Maconha altera a percepção que tenho das coisas (música, imagens, etc.) & & 0,688 & & & \\
\hline 7. Maconha ajuda-me a relaxar & 0,488 & & & & \\
\hline 8. Maconha prejudica a minha memória & & & & 0,803 & \\
\hline 9. Tenho mais prazer no sexo quando fumo maconha & & & 0,916 & & \\
\hline 10. É difícil controlar o meu desejo de fumar maconha & & & & & 0,708 \\
\hline 11. Maconha deixa-me disperso e com dificuldade de concentração & & & & 0,634 & \\
\hline 12. Quando fumo maconha vejo coisas que não existem (alucinações) & & 0,586 & & & \\
\hline 13. Maconha prejudica o meu aprendizado e/ou meu desempenho no trabalho & & & & 0,630 & \\
\hline 14. Fico mais feliz quando fumo maconha & 0,823 & & & & \\
\hline 15. Quando fumo maconha aproveito mais as festas e a diversão é maior & 0,716 & & & & \\
\hline 16. Fico depressivo e desapontado comigo mesmo quando fumo maconha & 0,617 & & & & \\
\hline 17. Maconha deixa-me mais interessado em sexo & & & 0,871 & & \\
\hline 18. Maconha ajuda-me a lidar com sentimentos ruins como angústia, ansiedade, tristeza & 0,562 & & & & \\
\hline
\end{tabular}


Inventário de Expectativas de Resultados em Usuários de Maconha - Pedroso et AL.

Tabela 3 - Coeficiente de correlação linear de Pearson entre as subescalas do IERUM

\begin{tabular}{|c|c|c|c|c|c|}
\hline & Aspectos emocionais & Percepção & Sexualidade & Aspectos cognitivos & Craving \\
\hline Aspectos emocionais & 1 & $0,403 \dagger$ & $0,452 \uparrow$ & 0,127 & $0,206 \dagger$ \\
\hline Percepcão & $0,403 \dagger$ & 1 & $0,286 \dagger$ & $0,422 \dagger$ & $0,316+$ \\
\hline Craving & $0,206 \dagger$ & $0,316 \dagger$ & 0,101 & $0,310 \dagger$ & 1 \\
\hline Aspectos cognitivos & 0,127 & $0,422 \dagger$ & 0,072 & 1 & $0,310 \dagger$ \\
\hline Sexualidade & $0,452 \uparrow$ & $0,286 \dagger$ & 1 & 0,072 & 0,101 \\
\hline
\end{tabular}

IERUM = Inventário de Expectativas de Resultados em Usuários de Maconha.

$* \mathrm{p}<0,05$.

$\dagger \mathrm{p}<0,01$.

Tabela 4 - Correlações de Pearson entre as subescalas do IERUM e as EAV

\begin{tabular}{lccccc}
\hline & Aspectos emocionais & Percepção & Sexualidade & Aspectos cognitivos & Craving \\
\hline EAV1 - percepção e cognição & 0,001 & $0,249^{*}$ & 0,044 & $0,406^{*}$ & $0,173 \dagger$ \\
EAV2 - sociabilidade & $0,217^{*}$ & 0,069 & $0,244^{*}$ & $-0,095$ & $-0,001$ \\
EAV3 - sexualidade & 0,105 & $0,248^{*}$ & $0,224^{*}$ & 0,078 & 0,021 \\
EAV4 - aspectos emocionais & 0,135 & $0,259^{*}$ & $0,336^{*}$ & $0,182 \dagger$ & 0,060 \\
EAV5 - craving & 0,107 & $0,283^{*}$ & 0,040 & $0,377^{*}$ & $0,517^{*}$ \\
\hline
\end{tabular}

EAV = escalas analógico-visuais; IERUM = Inventário de Expectativas de Resultados em Usuários de Maconha.

$* \mathrm{p}<0,01$

$\dagger \mathrm{p}<0,05$

Tabela 5 - Comparação pelo teste Mann-Whitney das subescalas do IERUM entre indivíduos dependentes e com uso nocivo de maconha

\begin{tabular}{lcccc}
\hline & \multicolumn{3}{c}{ Transtorno por uso de Cannabis } \\
\cline { 2 - 5 } Subescalas IERUM & Dependente $(\mathbf{n}=\mathbf{1 5 0})$ & Uso nocivo $(\mathbf{n}=\mathbf{3 1})$ & $\mathbf{U}$ & $\mathbf{p}$ \\
\hline Aspectos emocionais & $24,34(6,35)$ & $24,33(6,12)$ & 1325,00 & 0,934 \\
Percepção & $19,57(8,08)$ & $23,22(7,22)$ & 990,00 & 0,070 \\
Sexualidade & $7,64(4,68)$ & $8,67(4,83)$ & 1177,00 & 0,388 \\
Aspectos cognitivos & $14,46(5,84)$ & $16,89(4,13)$ & 1064,50 & 0,149 \\
Craving & $5,60(4,28)$ & $4,11(2,32)$ & 1127,00 & 0,247 \\
\hline
\end{tabular}

IERUM = Inventário de Expectativas de Resultados em Usuários de Maconha.

Expectativas de resultado frente ao uso da maconha As médias de pontos em cada subescala foram: 24,24 $(\mathrm{DP}=6,39 ; 5-35)$ em aspectos emocionais; 20,13 (DP = 8,11; 5-35) em percepção; 7,60 (DP = 4,70; 2-14) em sexualidade; $14,75$ (DP $=5,72 ; 3-21)$ em aspectos cognitivos; e 5,53 (DP $=4,16 ; 2-14)$ em craving. Se dividirmos essas médias pelo número de questões de cada subescala, obteremos: 4,85 de aspectos emocionais, 4,03 de percepção, 3,80 de sexualidade, 4,92 de aspectos cognitivos e 2,77 de craving.

\section{Discussão}

O IERUM demonstrou, após o processo de validação semântica, ser um instrumento adequado, de fácil e rápida aplicação e com bom nível de confiabilidade. Demonstrou também excelente equivalência semântica e conceitual, conforme os resultados do brainstorming ${ }^{10,11}$, sendo que todo o processo se fundamentou no estudo Pasquali ${ }^{9}$.
O valor do $\alpha$ de Cronbach da escala total de 17 itens (versão final do IERUM após análise fatorial) foi de 0,779 , o que é considerado por Rowland et al. ${ }^{20}$ como uma consistência interna plenamente satisfatória, pois o valor mínimo de 0,70 foi recomendado por considerar-se que os itens avaliam de modo consistente o mesmo construto. Ao comparar-se a consistência interna das subescalas, observaram-se variações nos resultados, que foram mais satisfatórios nas subescalas: craving, 0,69 $(\mathrm{n}=2)$, aspectos cognitivos, 0,69 $(\mathrm{n}=3)$ e sexualidade $0,83(\mathrm{n}=2)$.

A partir da análise fatorial houve a exclusão de um item (questão 4), pois ele estaria em dois fatores. Os 17 itens foram distribuídos em cinco subescalas: I. aspectos emocionais, II. percepção, III. aspectos cognitivos, IV. craving e V. sexualidade.

Quanto à validade do critério, verificou-se que a maior parte das correlações foram positivas e significativas, sem que houvesse redundância, conforme preconizam Ferrans \& Powers ${ }^{17}$. Não foram encontradas correlações somente entre 
as subescalas craving e sexualidade; aspectos emocionais e aspectos cognitivos; e aspectos cognitivos e sexualidade.

$\mathrm{Na}$ análise convergente, foram encontradas correlações positivas de intensidade de baixas a médias, destacando-se as correlações entre a subescala aspectos cognitivos do IERUM e a EAV percepção e cognição e entre a subescala craving do IERUM e a EAV craving. A correlação baixa da subescala percepção (IERUM) com a percepção e cognição (EAV) pode ter ocorrido devido a esta última abranger aspectos da subescala aspectos cognitivos. Foi encontrada correlação positiva, mas de baixa intensidade entre a subescala sexualidade (IERUM) e a sexualidade da EAV, e não foi encontrada correlação entre a subescala aspectos emocionais (IERUM) e aspectos emocionais (EAV), resultados estes que podem estar associados com a pouca especificidade/sensibilidade das $\mathrm{EAV}^{15}$.

$\mathrm{Na}$ análise discriminante, no entanto, a aplicação do IERUM não demonstrou haver diferenças significativas nas crenças de dependentes e usuários nocivos de maconha, podendo-se inferir que isso está relacionado ao fato de ambos os grupos terem feito uso da substância, tornando homogêneas as suas expectativas de resultados. Esse resultado difere do obtido na análise discriminante realizada na validação do $\mathrm{MEQ}^{5}$, que encontrou diferenças nos escores entre usuários e não usuários de maconha.

As médias mais altas por questão foram, em ordem decrescente, as das subescalas aspectos emocionais e aspectos cognitivos e percepção, o que está de acordo com os resultados de alguns estudos $^{5-7}$ que ressaltam esses itens como relacionados às expectativas dos efeitos da maconha. A menor média da subescala $\mathrm{cra}$ ving também foi observada no estudo de Pedroso et al. ${ }^{5}$.

Entender estas expectativas pode auxiliar o terapeuta a motivar os dependentes químicos a interromperem o uso da maconha ${ }^{1-6}$, servindo como um norteador do processo psicoeducacional. Não se deve esquecer que são essas expectativas positivas relacionadas aos efeitos de uma substância que servem como gatilho para o craving, podendo levar a lapsos ou recaídas ${ }^{2,16}$.

A construção de uma escala breve torna-se importante considerando que seu custo é reduzido e o tempo de aplicação permite a realização de pesquisas que podem contribuir estrategicamente para novos tratamentos, bem como seu uso durante consultas psicoterápicas.

Dentre as limitações deste estudo, cita-se o fato de o instrumento ter sido aplicado apenas em um grupo de sujeitos em que todos eram usuários de maconha (dependentes ou que faziam uso nocivo), o que interferiu nos resultados da análise discriminante. Outra limitação está associada ao perfil desses usuários, a maioria deles sujeitos internados, dependentes de outras substâncias psicoativas e que utilizavam uma quantidade significativa de cigarros de maconha por semana.
Espera-se que o IERUM seja um instrumento útil não só para os pesquisadores como para os profissionais que atendem dependentes químicos, auxiliando estes na avaliação das expectativas de resultados frente ao uso de maconha e tornando, assim, um pouco menos complicado o tratamento e a difícil manutenção da abstinência.

\section{Referências}

1. Eiser JR. Attitudes and beliefs. In: Baum A, McManus C, Newman S, Weinman J, West R, eds. Cambridge handbook of psychology, health and medicine. Cambridge: Cambridge University Press; 1997. p. 3-7.

2. Marlatt A, Gordon J. Prevenção da recaída: estratégia e manutenção no tratamento de comportamentos aditivos. Porto Alegre: Artes Médicas; 1993.

3. Pedroso RS, Oliveira MS, Araujo RB, Castro MG, Melo WV. Expectativas de resultados frente ao uso de álcool, maconha e tabaco. Rev Psiquiatr RS. 2006;28(2):198-206.

4. Brown SA. Drug effect expectancies and addictive behavior change. Exp Clin Psychopharmacol. 1993;1(1-4):55-67.

5. Stacy AW, Widaman KE, Marllat GA. Expectancy models of alcohol use. J Pers Soc Psychol. 1990;58(5):918-29.

6. Pedroso RS, Oliveira MS, Moraes JF. Tradução, adaptação e validação da versão brasileira da escala Marijuana Expectancy Questionnaire. Cad Saude Publica. 2007;23(1):63-73.

7. Hawkins LW. The impact of personality and effect on college student's motives for marijuana use. Dissertation Abstracts International: Section B: The-Sciencesand-Engineering. 2007;68:1306.

8. Stein LA, Colby S, Barnett N, Monti P, Lebeau-lebeau-Craven R, Golembeske C. Assessment of substance use and treatment implications for incarcerated teens. In: Eisenberg RM, Geller EB, eds. Proceedings from the 65th annual meeting of the college on problems of drug dependence. Bal Harbour; 2003.

9. Pasquali L. Princípios de elaboração de escalas psicológicas. Rev Psiquiatr Clin. 1998;25(5):206-13.

10. Pedroso RS, Oliveira MS, Araujo RB, Moraes JF. Tradução, equivalência semântica e adaptação cultural do Marijuana Expectancy Questionnaire (MEQ). Psico USF. 2004;9(2):129-36.

11. Duarte PS, Miyazaki MC, Ciconelli RM, Sesso R. Tradução e adaptação cultural do instrumento de avaliação de qualidade de vida para pacientes renais crônicos (KDQOL-SF TM). Rev Assoc Med Bras. 2003;49(4):375-81.

12. Organização Mundial de Saúde. Classificação de transtornos mentais e de comportamento da CID-10: descrições clínicas e diretrizes diagnósticas. Porto Alegre: Artmed; 1993.

13. Likert R. A technique for the measurement of attitudes. Arch Psychol. 1932;140(22):1-55.

14. Araujo RB, Oliveira MS, Moraes JF, Pedroso RS, Port F, Castro MG. Validação da versão brasileira do Questionnaire of Smoking Urges-Brief. Rev Psiquiatr Clin. 2007;34(4):166-75.

15. Araujo RB, Oliveira MS, Mansur MA. A validação brasileira do Questionnaire of Smoking Urges. Cad. Saude Publica. 2006;22(10):2157-67.

16. Araujo RB, Oliveira MS, Pedroso RS, Miguel AC, Castro MGT. Craving e dependência química: conceito, avaliação e tratamento. J Bras Psiquiatr. 2008;57(1):57-63.

17. Osborn AF. Applied imagination: principles and procedures of creative thinking. New York: Charles Scribners Sons; 1953.

18. Tiffany ST, Drobes DJ. The development and initial validation of a questionnaire on smoking urges. Br J Addict. 1991;86(11):1467-76.

19. Ferrans CE, Powers MJ. Psychiatric assessment of the Quality of Life Index. Res Nurs Health. 1992;15:29-38.

20. Rowland D, Arkkelin D, Crisler L. Computer-based data analysis:using SPSSXI in the Social and Behavioral Sciences. Chicago: Nelson-Hall; 1991. 